Proceedings

\title{
On the Informational Essence of Emergence and Evolution: An Analysis of the New Dualistic Approach ${ }^{\dagger}$
}

\author{
Ping Wang and Jian Wang * \\ School of Humanities and Social Science, Xi'an Jiaotong University, Xi'an, China; phoebe0805@126.com \\ * Correspondence: wangjian0501@sina.com; Tel.: +86-150-9403-0651 \\ + Presented at the IS4SI 2017 Summit DIGITALISATION FOR A SUSTAINABLE SOCIETY, Gothenburg, \\ Sweden, 12-16 June 2017.
}

Published: 8 June 2017

\begin{abstract}
In his book "What is Information", Robert Logan explores certain issues related to information on the basis of the connotation of information itself, and puts forward two important theories of "extended mind" and "symbolosphere". Based on the strong-emergence theory, Logan depicts the material emergence and non-material emergence, and proposes a neo-dualism view, a weak form of dualism. According to this, different from the biosphere, the evolution and reproduction mechanism in symbolosphere do not follow the rules of genetic inheritance, but the mechanisms of memes, belonging to the territory of information study. However, the new dualism faces the difficulty to correctly explain the ontological position of the symbolosphere, while the philosophy of information provides a standard solution in its theory of human evolution.
\end{abstract}

Keywords: neo-dualism; emergence; information

\section{Introduction}

In his book "What is Information", Robert K. Logan explores his interest in the meaning of information and how does the understanding of information affect human beings. By reviewing the history of ideas about information, from Hartley, Shannon, Weaver and others to Gordon Mackay and Gregory Bateson, Logan takes his mission to find those necessary elements of information in this series of exploration that may be excluded, and to recognized a number of distinct and advanced concepts of information.

Clearly, there are two resources which inspire Logan on his attempt to understand information and its applications in the information age. One is from achievements of Marshall McLuhan. Robert Logan dives into the deeper aspects of the problem of information due to his collaborations with McLuhan on topics like the origins of writing and the nature of medium, etc. It is fair to say that from the general communication approach, Logan starts his exploration into the information issue. And the other is from system theory scholar Stuart Kauffman. The key concept and vital argument element in this paper is the propagation of organization, and how does it show its distinctiveness in the biosphere, the symbolosphere, the technosphere and the econosphere. And the notion that information is always dependent on constraints is thus promoted on this basis of theory, which also constitutes the foundation of argument for the rest of his book.

On the foreword for this book, Terrence Deacon points out that a synthesis of two ways of conceiving of human thought and communication are the core insight that runs through the book. The first insight is that mind-at least human mind - is not a phenomenon that is confined within a brain. The "extended mind" hypothesis argues that human minds are integrated with the sea of communication it is embedded within. Thus no mind is an island. The second insight is that this web of communication in many important respects exists and evolves and exhibits causal dynamics that 
persist in parallel with the physical-mechanical-biological world. This domain of meanings, purposes and values has been called the "Symbolosphere" by John Schumann [1]. These two crucial insight intertwine naturally in Logan's book and further improve his explorations of information.

\section{Emergence and Neo-Dualism}

\subsection{Material and Non-Material Emergence}

Although human symbolic interactions are naturally part of the human biotic system, Logan makes distinction between the so-called purely biological interactions of biosemiosis and human language/culture. While in the former process, information is not standing for something else and the communication of information is instantiated in the biomolecules, and cannot be separated from those biomolecules, in the latter process, information is clearly symbolic and is not instantiated materially but is only physically mediated, therefore human language and culture are able to move from one medium to another. Based on these, Logan states that the distinction between material and non-material emergence can be made. The familiar examples of material emergence are regular hexagonal convection cells, weather patterns in the abiotic world and living organism in the biosphere. And for non-material emergence, it could be included into the symbolosphere as Schumann originally introduced [1], which involves the human mind and all the products of the mind, like its abstract thoughts and symbolic communication processes such as spoken and written language and the other products of the human mind and culture such as music, art, math, science and technology.

As Kauffman [2] and Clayton [3] argue that biology cannot be predicted from or reduced to physics, the symbolic conceptual non-material aspects of human behavior cannot be reduced to, derived from or predicted from the biology of human brain and the nervous system. Human language and culture as the symbolic domain are a product of human conceptual thought and represent emergent phenomena and propagating organization, symbolic and not materially instantiated. There is an exception of technology, which seems be instantiated in physics, but it is the concepts and organization that goes in to the creation of physical tools which are emergent and propagate not the actual physical tools.

\subsection{New Dualism}

The notion of neo-dualism was firstly introduced in Logan and Schumann and extended in Logan [4], due to the existence of non-material emergence and the symbolosphere. Different from the definition of traditional dualism as in Clayton [3], the non-physical component to humans namely language, culture and mind is symbolic and conceptual, not necessarily spirit-like or transcendent. In this neo-dualistic approach of Logan and Schumann [4], all phenomena belong to one or the other of two different domains: the physiosphere, the material world consisting of both living and non-living matter and corresponding exactly to Descartes' res extensa, and the symbolosphere, involving the human mind and all the symbolic products of the mind and corresponding to Descartes' res cogitans minus the notion of God, the soul and spirit. In this way, neo-dualism represents a weak form of dualism, contrasted with the strong dualism of Descartes.

Because the symbolosphere cannot be reduced to the biology of the human brain and the nervous system from which they arise, Logan sticks to the strong emergence position, instead of that of the weak emergence which confirms the distinction between the higher levels of complexity and lower levels but holds the view that higher level phenomena can ultimately be reduced to physics once a deeper understanding of the world is gained. In this case, the strong emergence and neo-dualism/weak dualism are perfectly compatible.

\section{Informational Nature in the Evolution and Propagation of Symbolosphere}

As pointed by Deacon, human beings are symbolic species, elements of the symbolosphere are exactly the behaviors of human race. And it is also the symbolic element that unite all other elements in the symbolosphere. 
The notion of weak dualism or neo-dualism sheds light on our understanding of propagating organization and the non-material emergence. The differences lay in that it is the material gene-based propagating organization in biosphere while it is non-material, extra-somatic, meme-based propagating organization in the symbolosphere. Just as the mechanism for replication for living organisms through descent, modification and selection, the same is true of the evolution of the symbolosphere. The transmission of meme is its descent, though varied in forms and levels. At the same time, because of the transmitting ability of meme differs and modification of memes in minds of various recipients, the selection process occurs along with. Thus, we could see that the propagating organization of the symbolosphere in nature follows an evolution mechanism of information, and the symbolosphere belongs to the domain of information.

\section{Insight from the Philosophy of Information}

Logan's interpretation of human culture and society focuses on the emergence and downwards causal relations. He depicts the mode of propagation and evolution in the symbolosphere. However, the new dualism faces the difficulty to correctly explain the ontological position of the symbolosphere, while the philosophy of information proposed by Chinese scholar Kun Wu provides a standard solution in its theory of human evolution [5]. The evolution of human beings not only contains a physiological inheritance pattern, that is, to follow the single evolution path with DNA genetic characteristics, but also includes psychological activity patterns and behavioral patterns in a three-dimensional way. For human race, the physiological and genetic characteristics will present themselves in the postnatal growth, at the same time, the characteristics of psychological and behavior patterns accumulated in years will also leave "traces" on the inherent genetic vector, which constitutes a new congenital genetic features. It is in this interaction and two-way activities of mutual development and realization between human and nature as well as culture factors that all the content of physiosphere, biosphere and symbolosphere and their form achieve a completed, essential and unified integration.

In this book, Logan have clearly demonstrated that many serious limitations exist in our current understanding of information. His efforts, that information should be perceived in a larger theory of the "propagating organization", rather than the reproduction and transmission of bits of data, paves the way to explore pragmatic aspects of symbolic communication of human beings. It will bring inspiring insight to our comprehension and illustration of symbolic evolution of society in digital age, from science to technology and from computation to Internet. And the philosophy of information, on the other hand, offers us a systematic approach to understand the dual existence of matter and information in the ontological sense.

Conflicts of Interest: The authors declare no conflict of interest.

\section{References}

1. Schumann, J.H. The evolution of the symbolosphere. In Proceedings of the Great Ideas in the Social Sciences Lecture, UCLA Center for Governance, Los Angeles, CA, USA, 21 November 2003.

2. Kauffman, S. Investigations; Oxford University Press: Oxford, UK, 2000.

3. Clayton, P. Mind and Emergence: From Quantum to Consciousness; Oxford University Press: Oxford, UK, 2004.

4. Logan, R.K. Neo-dualism and the bifurcation of the symbolosphere into the mediasphere and the human mind. Semiotica 2006, 160, 229-242.

5. Wu, K. Philosophy of Information: Theory, Method and System; The Commercial Press: Beijing, China, 2005; pp. 266-275.

(C) 2017 by the authors. Licensee MDPI, Basel, Switzerland. This article is an open access article distributed under the terms and conditions of the Creative Commons Attribution (CC BY) license (http://creativecommons.org/licenses/by/4.0/). 